\title{
The Role of Geriatric Nutritional Risk Index in Predicting Postoperative Pulmonary Complications in Elderly Lung Cancer Patients Undergoing Surgical Resection
}

\author{
Mao Xiaowei \\ Shanghai Jiao Tong University \\ Zhang Wei \\ Shanghai Jiao Tong University \\ Hu Fang \\ American-Sino Women's \& Children's Hospital \\ Niu Yanjie \\ Shanghai Jiao Tong University \\ Wang Qiang \\ Taizhou central hospital \\ Lu Ai-ting \\ Shanghai Jiao Tong University \\ Liu Jiabing \\ Shanghai Jiao Tong University \\ Jiang Liyan ( $\nabla$ jiang_liyan2000@126.com ) \\ Shanghai Jiao Tong University
}

\section{Research Article}

Keywords: geriatric nutritional risk index, postoperative pulmonary complication, lung cancer, resection, predictor.

Posted Date: January 5th, 2022

DOI: https://doi.org/10.21203/rs.3.rs-1155133/v2

License: (c) (1) This work is licensed under a Creative Commons Attribution 4.0 International License. Read Full License 


\section{Abstract \\ Background}

The relationship between immunonutritional status (eg. Prognostic nutritional index [PNI] and Controlling Nutritional Status [COUNT] score) and risk of postoperative pulmonary complications (PPCs) after surgical resection of lung cancer had reported before. However, another immunonutritional parameterGeriatric Nutritional Risk Index (GNRI)-had never explored.

\section{Method}

To address this issue, in this study we retrospectively analyzed patients' characteristics and PPCs in a cohort of lung cancer patients who were treated by surgical resection at our center. The clinical utility of patients' characteristics for predicting PPCs was evaluated by receiver operating characteristic curve analysis and the Youden index. Univariate and multivariate analysis were applied to find the most important factors.

\section{Result}

A total of 128 patients met the inclusion criteria for this study. Significant differences in sex, GNRI, FEV1\%, LY\% were found between the PPC and non-PPC groups (all $\mathrm{P}<0.05$ ). The difference in pathology between the 2 groups showed borderline statistical significance $(P=0.052)$. We determined the best cutoff value of each parameter and calculated the corresponding sensitivity and specificity, and found that GNRI, FEV1\% and LY\% had similar diagnostic value. Multivariate analysis reveled GNRI, sex, LY\% and FEV1\% were filtered to be correlated to PPCs of elderly lung cancer patients received surgery therapy.

\section{Conclusion}

These results indicate that preoperative immunonutritional parameters of GNRI can be used to identify elderly lung cancer patients at risk of PPCs.

\section{Introduction}

Lung cancer is the leading cause of cancer-related deaths both worldwide and in China ${ }^{1,2}$. Surgery is the first-choice treatment for early-stage lung cancer ${ }^{3}$. The mortality and postoperative pulmonary complication (PPC) rates within 30 days after lung cancer resection are about $4.4 \%$ and $20-40 \%$, respectively ${ }^{4,5}$. Many factors influence the occurrence of PPCs, including preoperative pulmonary function and immunonutritional status ${ }^{6,7}$. 
The immunonutritional status of lung cancer patients were an important predictor of clinical outcome. Tumor cells alter the patient's metabolism and grow rapidly by usurping nutrients from the patient. It was previously reported that weight loss and low albumin (ALB) level were associated with the occurrence of PPCs after lung cancer resection ${ }^{8}$. Various instruments such as modified Glasgow prognostic score (mGPS), prognostic nutritional index (PNI), Controlling Nutritional Status (CONUT) score, and Geriatric Nutritional Risk Index (GNRI) have been developed to assess the preoperative immunonutritional status of cancer patients. The relationship between immunonutritional status (eg. PNI, CONUT score) and risk of PPCs after surgical resection of lung cancer had reported before ${ }^{9,10}$. However, another immunonutritional parameter- GNRI -had never explored.

To address the above point, in this retrospective cohort study we evaluated the clinical utility of GNRI for predicting PPCs in elderly lung cancer patients treated by surgical resection.

\section{Methods}

\section{Patient selection}

We reviewed the patient database of Shanghai Chest Hospital, Shanghai Jiao Tong University for lung cancer cases treated by resection in 2012 and 2013. The study was conducted according to the principles outlined in the Declaration of Helsinki, and the Ethics Committee of Shanghai Chest Hospital approved the protocol (no. KS1924). All patients signed the informed consent form before participating.

The inclusion criteria were as follows: (1) age between 60 and 80 years old; (2) underwent lung cancer resection at Shanghai Chest Hospital, Shanghai Jiao Tong University; (3) physical and laboratory examinations were performed within 1 week before the surgery. Exclusion criteria were as follows: (1) incomplete clinical data; (2) severe cardiac insufficiency as determined by electrocardiography and echocardiography; (3) previously underwent lung resection. Basic demographic and clinical information were collected for each patient including age, sex, smoking history, physical and laboratory examination results, major comorbidities, surgery type, pathologic diagnosis, and PPCs.

\section{Evaluation of immunonutritional status}

A standard physical examination was conducted and data including height and weight were recorded. Laboratory examinations were also performed before surgery including a routine blood test, hepatic and renal function tests, and measurement of serum lipids. The immunonutritional parameters were determined as follows.

GNRI was calculated based on serum ALB level and body weight according to the formula GNRI=1.489 ×serum ALB level $(\mathrm{g} / \mathrm{L})+41.7 \times$ preoperative weight/ideal weight $(\mathrm{kg})^{11}$. 


\section{Surgery performance}

Four surgeons and their respective teams performed all operations, using similar operative and perioperative procedures. All patients received similar medical care following the guidelines of enhanced recovery after surgery ${ }^{12}$. Preoperative medical care included health education, smoking cessation, preoperative pulmonary rehabilitation, comorbidities management, and prevention of deep venous thrombosis. Intraoperative management included general anesthesia combined with endotracheal intubation anesthesia and paravertebral nerve block under ultrasound guidance; precise controlled infusion of opioid frugal general anesthesia technology was used to reduce the dosage of muscle relaxants. Postoperative management included post-operative pulmonary rehabilitation and nutritional support. Each patient was given prophylactic antibiotics for 2-3 days after surgery.

\section{Evaluation of PPCs}

PPCs within 1 month after lung cancer resection were defined as follows: (1) pneumonia (new lung infiltration on imaging examination in addition to any of the following items: fever [body temperature $\geq 38^{\circ} \mathrm{C}$ ] lasting $>5$ days; white blood cell count $>10.5 \times 10^{9} / \mathrm{l}$; and upgraded antibiotic treatment or antibiotic application for $>7$ days); (2) atelectasis; (3) long-term mechanical ventilation due to postoperative respiratory failure (mechanical ventilation time $>48 \mathrm{~h}$ ); (4) secondary tracheal intubation; (5) persistent air leakage or pleural effusion requiring long-term drainage (>7 days); (6) bronchopleural fistula; (7) pulmonary embolism; (8) other pulmonary complications ${ }^{13,14}$.

\section{Statistical analysis}

We used SPSS v20.0 software (IBM, Armonk, NY, USA) for statistical analyses. Measurement data are expressed as mean $\pm S D$. Differences between groups were evaluated by one-way analysis of variance and with the $t$ test. Count data were analyzed with Pearson's chi-squared test and Fisher's exact probability test. Receiver operating characteristic (ROC) curve analysis and the Youden index were used to assess the predictive value of immunonutritional parameters for PPCs. Differences were considered statistically significant for $P$ values $<0.05$.

\section{Results}

\section{Characteristics of the study population}

A total of 128 patients met the inclusion criteria for this study (Fig. 1). Most patients were male (73.43\%), underwent thoracotomy (93.75\%), had no smoking history (45.31\%), and were diagnosed with adenocarcinoma (54.69\%) (Table 1). There were 29 patients with 37 PPCs in the whole cohort, the 
incidence of PPCs was 22.48\% (29/128). The most frequent PPCs were persistent air leakage or pleural effusion and pneumonia. Six patients had more than one PPC (Table 2).

\section{Relationship between immunonutritional status and PPCs}

We calculated the correlation between patient characteristics and PPCs. Significant differences in sex, GNRI, FEV1\%, LY\% were found between the PPC and non-PPC groups (all $P<0.05$ ). The difference in pathology between the 2 groups showed borderline statistical significance $(P=0.052)$. (Table 3$)$

\section{Predictors of PPCs}

An ROC curve analysis was performed with GNRI, BMI, FEV1\%, FVC and LY\% to evaluate the predictive value of these parameters for PPCs. GNRI, FEV1\%, FVC and LY\% were found to be statistically significant based on the area under the ROC curve. We determined the best cutoff value of each parameter and calculated the corresponding sensitivity and specificity, and found that GNRI, FEV1\% and LY\% had similar diagnostic value. (Table 4 and Fig. 2). The we conducted the multivariate analysis. For the continuous variable, we used the cutoff value to define "low" or "high". In the analysis, the variable of sex, surgery method, pathology, GNRI FEV1\%, FVC\% and LY\% were included. Finally, GNRI, sex, LY\% and FEV1\% were filtered to be correlated to PPCs of elderly lung cancer patients received surgery therapy.

\section{Discussion}

PPCs are a major concern in the surgical treatment of lung cancer. Pulmonary function is one of the main predictors of PPCs, and some guidelines recommend the evaluation of preoperative immune and nutritional status ${ }^{15}$. The PPC rate in our study is about $22 \%$, which is similar to previous reports ${ }^{4,5}$. The main PPC is persistent air leakage or pleural effusion and pneumonia, which are affected by immune and nutritional status. Pneumonia is common in patients who are malnourished or immunocompromised. The principal causes of persistent air leakage or pleural effusion are pneumothorax or hydrothorax; persistent hydrothorax may also result from pneumonia or hypoproteinemia. Thus, in theory, immune and nutritional parameters can be predictors of PPCs.

Many researches have explored the clinical meanings of single immune and nutritional in predicting PPCs. Among all, ALB and body weight related index are most studied. ALB is synthesized by hepatocytes and maintains vascular osmotic pressure; transports hormones, fatty acids, or other compounds; and regulates blood pH. Serum ALB concentration is often used as a biochemical marker of long-term nutritional status and reflects visceral protein status ${ }^{16}$. A correlation between ALB insufficiency and the development of early PPCs has been reported previous ${ }^{17,18}$. Body weight loss is also revealed correlated to high rate of PPCs. In Busch E's report, weight loss and ALB level are linked to PPCs following lung cancer resection ${ }^{8,19-21}$. Globulin and $L Y \%$ reflect the immune status of patients ${ }^{22}$. The cellular 
immunitymainly regulates immune function through $\mathrm{T}$ lymphocyte subsets ${ }^{23}$ and in tumor surveillance, immunoglobulin contributes to target cell phagocytosis ${ }^{24}$.

Immunonutritional parameters are calculated based on multiple indices related to immune function (CRP, peripheral lymphocyte count, etc) and nutrition (BMI and ALB). Those parameters show to be useful for detecting sarcopenia, which has been linked to the survival of lung cancer patients ${ }^{25}$. Moreover, several studies have reported that immunonutritional parameters are prognostic indicators of survival ${ }^{26-33}$ and could predict PPCs ${ }^{28,30,34}$ in patients with lung cancer. Poor immunonutritional status may increase the risk of PPCs by impairing pulmonary function, which was improved by nutritional support during chemotherapy ${ }^{33}$.

Among the clinical instruments included in our analysis, GNRI were found to have predictive value for PPCs, with moderate diagnostic value. GNRI are calculated based on the original index and are continuous variables. In previous study, GNRI is suggested to be not inferior to nutrition screening tool of Mini Nutritional Assessment ${ }^{35}$. GNRI is recognized to correlated with sarcopenia status ${ }^{36}$. GNRI has been widely applied in digestive tract diseases. In gastric cancer, colorectal cancer and esophageal cancer, GNRI is defined as a prognostic factor ${ }^{37-39}$. Previous studies reveal GNRI as a prognostic factor both in early lung cancer patients treated with surgery operation ${ }^{40,41}$ and advanced stage lung cancer ${ }^{42-44}$. Besides, GNRI can predict the treatment response of immunotherapy ${ }^{45,46}$. Apart this, GNRI is suggested to be good predictor for postoperative complications after abdominal surgery or gastrointestinal malignancy ${ }^{47,48}$. But, can this parameter be good predictor in elderly lung cancer surgery operation patients? There is no answer. Thus we compare the GNRI and other patients characteristic and find GNPI has moderate sensitivity for detecting PPCs; that is, patients with low values are more likely to develop PPCs after lung cancer resection and require special care. Meanwhile, it has moderate specificity; this means that patients with a high value are not likely to experience PPCs, and routine care is therefore sufficient. Thus, risk stratification is possible based on these few parameters, which would maximize the use of limited medical resources. As mention above, ALB and body weight are the most important simple index for PPCs. When calculating GNRI, both ALB and body weight are enrolled, thus it may has excellent diagnostic value than other patient characteristics.

There were some limitations to this study that should be noted. Firstly, because of the retrospective single-center design, selection bias could not be avoided. Secondly, because the sample size is relatively small, statistical bias was inevitable.

Nonetheless, our findings demonstrate that immunonutritional parameters can predict PPCs following lung cancer resection and can be used to identify high-risk patients who would benefit from preventive interventions. Among all, GNRI has the best performance.

\section{Abbreviations}

PPC: postoperative pulmonary complication 

ALB: albumin

mGPS: modified Glasgow prognostic score

PNI: prognostic nutritional index

CONUT: controlling nutritional status

GNRI: geriatric nutritional risk index

ROC: receiver operating characteristic

VATS: video-assisted thoracoscope

BMI: body mass index

FEV1: forced expiratory volume

FVC: forced vital capacity

LY: lymphocyte

RBC: red blood cell

PALB: Prealbumin.

\section{Declarations}

\section{Ethics approval and consent to participate}

The study was conducted according to the principles outlined in the Declaration of Helsinki, and the Ethics Committee of Shanghai Chest Hospital approved the protocol (no. KS1924). All patients signed the informed consent form before participating.

\section{Consent for publication}

All author approved this publication.

\section{Availability of data and materials}

The datasets generated for this study will be made available from the corresponding author on reasonable request.

\section{Competing interests}

The authors declare that the research was conducted in the absence of any commercial or financial relationships that could be construed as a potential conflict of interest. 


\section{Funding}

This work was support by grants from the National Key Research and Development Program of China (no. 2018YFC1313600 to LY Jiang) and Chinese Society of Clinical Oncology (no. Y-2019AZZD-0038 to LY Jiang).

\section{Author Contributions}

Conception and design: Mao XW, Zhang W, Jiang LY; (II) Administrative support: Mao XW, Jiang LY; (III) Provision of study materials or patients: Mao XW, Zhang W, Hu F, Niu YJ, Jiang LY; (IV) Collection and assembly of data: Mao XW, Zhang W, Hu F, Wang Q, Lu AT, Liu JB; (V) Data analysis and interpretation: Mao XW, Zhang W, Hu F, Wang Q, Lu AT, Liu JB, Jiang LY; (VI) Manuscript writing: All authors; (VII) Final approval of manuscript: All authors

\section{Acknowledgements: None}

\section{Authors' information:}

Mao Xiaowei: 1. Pulmonary and Critical Care Medicine, Shanghai Jiao Tong University, Shanghai Chest Hospital, Shanghai, Chinaj. 2. Department of Pulmonary and Critical Care Medicine, Regional medical center for National Institute of Respiratory Diseases, Sir Run Run Shaw Hospital, School of Medicine, Zhejiang University, Hangzhou, China. E-mail: maoxwres@163.com.

Zhang Wei: 1. Pulmonary and Critical Care Medicine, Shanghai Jiao Tong University, Shanghai Chest Hospital, Shanghai, China. 2. Department of Internal Medicine, American-Sino Women's \& Children's Hospital, Shanghai, China. E-mail: zhwei61@sina.com.

Hu Fang: 1. Pulmonary and Critical Care Medicine, Shanghai Jiao Tong University, Shanghai Chest Hospital, Shanghai, China. E-mail: HuFang0527@163.com.

Niu Yanjie: 1. Pulmonary and Critical Care Medicine, Shanghai Jiao Tong University, Shanghai Chest Hospital, Shanghai, China. E-mail: lilynew@163.com.

Wang Qiang: 1. Department of thoracic surgery, Taizhou central hospital, Taizhou, Zhejiang Province, China. E-mail: wangq9414@tzzxyy.com.

Lu Ai-ting: 1. Department of Nursing, Shanghai Jiao Tong University, Shanghai Chest Hospital, Shanghai, China. E-mail: writing_lu@126.com

Liu Jiabing: 1. Department of radiotherapy, Shanghai Jiao Tong University, Shanghai Chest Hospital, Shanghai, China. E-mail: ljb_sjtu@163.com.

Jiang Liyan: 1. Pulmonary and Critical Care Medicine, Shanghai Jiao Tong University, Shanghai Chest Hospital, Shanghai, China. E-mail: jiang_liyan2000@126.com. 


\section{References}

1. International Agency for Research on Cancer. World Health Organization. Latest global cancer data: Cancer burden rises to 19.3 million new cases and 10.0 million cancer deaths in 2020. Questions and answers (Q\&A). Available at: https://www.iarc.fr/faq/latest-global-cancer-data-2020-qa/. Accessed March 3, 2021.

2. Chen W, Zheng R, Baade PD, et al. Cancer statistics in China, 2015. CA Cancer J Clin. 2016, 66(2): 115-132.

3. Baltayiannis N, Chandrinos M, Anagnostopoulos D, et al. Lung cancer surgery: An up to date. J Thorac Dis. 2013, Suppl 4 (Suppl 4): S425-439.

4. Radu DM, Jauréguy F, Seguin A, et al. Postoperative pneumonia after major pulmonary resections: An unsolved problem in thoracic surgery. Ann Thorac Surg. 2007, 84(5): 1669-1673.

5. Strand TE, Rostad H, Damhuis RA, et al. Risk factors for 30-day mortality after resection of lung cancer and prediction of their magnitude. Thorax. 2007, 62 (11): 991-997.

6. Tabutin M, Couraud S, Guibert B, et al. Completion pneumonectomy in patients with cancer: Postoperative survival and mortality factors. J Thorac Oncol. 2012, 7 (10):1556-1562.

7. Zhao S, Guo YQ, Zhu JQ, et al. Analysis of preoperative pulmonary function and postoperative quality of life of lung cancer patients. Journal of Clinical Pulmonary Medicine. 2014, 19 (3): 450452.

8. Busch E, Verazin G, Antkowiak JG, et al. Pulmonary complications in patients undergoing thoracotomy for lung carcinoma. Chest. 1994, 105 (3): 760-766.

9. Asakawa A, Ishibashi H, Matsuyama $Y$, et al. Preoperative nutritional status is associated with the prognosis for lung cancer. Asian Cardiovasc Thorac Ann. 2021, 29(8): 763-771.

10. Takahashi M, Sowa T, Tokumasu $\mathrm{H}$, et al. Comparison of three nutritional scoring systems for outcomes after complete resection of non-small cell lung cancer. J Thorac Cardiovasc Surg. 2021, 162(4): 1257-1268.e3.

11. Bouillanne O, Morineau G, Dupont C, et al. Geriatric Nutritional Index: A new index for evaluating atrisk elderly medical patients. Am J Clin Nutr. 2005, 82 (4): 777-783.

12. Cheng YJ, Hou XM, Xu TT, et al. Research of the effectiveness of thoracic surgery enhanced recovery practice in the improvement of medical value. Chinese Hospital Management. 2021; 41(4): 56-59.

13. Glogowska O, Glogowski M, Szmit S. Intensive rehabilitation as an independent determinant of better outcome in patients with lung tumors treated by thoracic surgery. Arch Med Sci. 2017, 13 (6):14421448.

14. Lai YT, Huang J, Yang M, et al. Seven-day intensive preoperative rehabilitation for elderly patients with lung cancer: A randomized controlled trial. J Surg Res. 2017, 209:30-36.

15. British Thoracic Society; Society of Cardiothoracic Surgeons of Great Britain and Ireland Working Party. BTS guidelines: Guidelines on the selection of patients with lung cancer for surgery. Thorax. 2001, 56 (2): 89-108. 
16. D'Angio RG. Is there a role for albumin administration in nutrition support? Ann Pharmacother. 1999, 28 (4): 478-482.

17. Zhao $\mathrm{K}$, Zhang J, Li J, et al. Incidence and risk factors of surgical site infection after intertrochanteric fracture surgery: A prospective cohort study. Int Wound J. 2020, 17(6): 1871-1880.

18. Jiang SP, Li ZY, Huang LW, et al. Multivariate analysis of the risk for pulmonary complication after gastrointestinal surgery. World J Gastroenterol. 2005, 11(24): 3735-3741.

19. Jagoe RT, Goodship TH, Gibson GJ. The influence of nutritional status on complications after operations for lung cancer. Ann Thorac Surg. 2001, 71 (3): 936-943.

20. Ahsan M. Arozullah, Shukri F. Khuri, et al. Development and validation of a multifactorial risk index for predicting postoperative pneumonia after major noncardiac surgery. Ann Intern Med. 2001, 135(10): 847-857.

21. Matsuoka K, Yamada T, Matsuoka T, et al. Significance of body mass index for postoperative outcomes after lung cancer surgery in elderly patients. World J Surg. 2018, 42 (1):153-160.

22. Egen JG, Ouyang WJ, et al. Human anti-tumor immunity: Insights from immunotherapy clinical trials. Immunity. 2020, 52 (1): 36-54.

23. Yeo KP, Angeli V. Bidirectional crosstalk between lymphatic endothelial cell and $\mathrm{T}$ cell and its implications in tumor immunity. Front Immunol. 2017, 8: 83.

24. Nag M, Bera K, Basak S. Intermolecular disulfide bond formation promotes immunoglobulin aggregation: Investigation by fluorescence correlation spectroscopy. Proteins. 2015, 83 (1): 169-177.

25. Fumihiro $S$. Clinical impact of preoperative immunonutritional status in patients undergoing surgical resection of lung cancer. J Thorac Dis. 2019, 11 (Suppl 3): S408-412.

26. Jin J, Hu KJ, Zhou YZ, et al. Clinical utility of the modified Glasgow prognostic score in lung cancer: A meta-analysis. PLoS One. 2017, 12 (9): e0184412.

27. Grose D, Morrison DS, Devereux G, et al. The impact of comorbidity upon determinants of outcome in patients with lung cancer. Lung Cancer. 2015, 87 (2): 186-192.

28. Okada S, Shimada J, Kato D, et al. Clinical significance of prognostic nutritional index after surgical treatment in lung cancer. Ann Thorac Surg. 2017, 104 (1): 296-302.

29. Jiang AM, Zhao R, Liu N, et al. The prognostic value of pretreatment prognostic nutritional index in patients with small cell lung cancer and its influencing factors: A meta-analysis of observational studies. J Thorac Dis. 2020, 12 (10): 5718-5728.

30. Lee SC, Lee JG, Lee SH, et al. Prediction of postoperative pulmonary complications using preoperative controlling nutritional status (CONUT) score in patients with resectable non-small cell lung cancer. Sci Rep. 2020, 10 (1):12385.

31. Miura N, Shoji F, Kozuma Y, et al. Preoperative immune-nutritional abnormality predicts poor outcome in elderly non-small-cell lung cancer patients with comorbidities. Ann Thorac Cardiovasc Surg. 2020, 26 (5): 240-247. 
32. Shoji F, Miura N, Matsubara T, et al. Prognostic significance of immune-nutritional parameters for surgically resected elderly lung cancer patients: A multicenter retrospective study. Interact Cardiovasc Thorac Surg. 2018, 26 (3): 389-394.

33. Hino $\mathrm{H}$, Saito $\mathrm{T}$, Matsui $\mathrm{H}$, et al. Utility of geriatric nutritional risk index in patients with lung cancer undergoing surgery. Eur J Cardiothorac Surg. 2020, 58 (4): 775-782.

34. Zhou PH, Zhang JF. Effects of parenteral nutrition support on immune function and lung function of patients with lung cancer after chemotherapy. Hebei Medical Journal. 2020, 42 (7): 2598-2601.

35. Abd-El-Gawad WM, Abou-Hashem RM, El Maraghy MO, et al. The validity of geriatric nutrition risk index: simple tool for prediction of nutritional-related complication of hospitalized elderly patients. Comparison with Mini Nutritional Assessment. Clin Nutr. 2014, 33(6): 1108-1116.

36. Shoji F, Matsubara T, Kozuma Y, et al. Relationship between preoperative sarcopenia status and immuno-nutritional parameters in patients with early-stage non-small cell lung cancer. Anticancer Res. 2017, 37(12): 6997-7003.

37. Naoshi Kubo, Katsunobu Sakurai, Tatsuro Tamura, et al. The impact of geriatric nutritional risk index on surgical outcomes after esophagectomy in patients with esophageal cancer. Esophagus. 2019, 16(2): 147-154.

38. Sasaki M, Miyoshi N, Fujino S, et al. The geriatric nutritional risk index predicts postoperative complications and prognosis in elderly patients with colorectal cancer after curative surgery. Sci Rep. 2020, 10(1): 10744.

39. Hirahara N, Tajima Y, Fujii Y, et al. Prediction of postoperative complications and survival after laparoscopic gastrectomy using preoperative geriatric nutritional risk index in elderly gastric cancer patients. Surg Endosc. 2021, 35(3): 1202-1209.

40. Shoji F, Matsubara T, Kozuma Y, et al. Preoperative geriatric nutritional risk index: A predictive and prognostic factor in patients with pathological stage I non-small cell lung cancer. Surg Oncol. 2017, 26(4): 483-488.

41. Hino $\mathrm{H}$, Saito $T$, Matsui $H$, et al. Utility of geriatric nutritional risk index in patients with lung cancer undergoing surgery. Eur J Cardiothorac Surg. 2020, 58(4): 775-782.

42. Peng SM, Yu N, Ren JJ, et al. The Geriatric Nutritional Risk Index as a prognostic factor in patients with advanced non-small-cell lung cancer. Nutr Cancer. 2020: 1-10.

43. Lee GW, Go SI, Kim DW, et al. Geriatric nutritional risk index as a prognostic marker in patients with extensive-stage disease small cell lung cancer: Results from a randomized controlled trial. Thorac Cancer. 2020, 11 (1): 62-71.

44. Matsuura S, Morikawa $\mathrm{K}$, Ito $\mathrm{Y}$, et al. The geriatric nutritional risk index and prognostic nutritional index predict the overall survival of advanced non-small cell lung cancer patients. Nutr Cancer. 2021: $1-8$.

45. Karayama $\mathrm{M}$, Inoue $\mathrm{Y}$, Yoshimura $\mathrm{K}$, et al. Association of the geriatric nutritional risk index with the survival of patients with non-small cell lung cancer after nivolumab therapy. J Immunother. 2021, Online ahead of print. 
46. Sonehara K, Tateishi K, Araki T, et al. Prognostic value of the geriatric nutritional risk index among patients with previously treated advanced non-small cell lung cancer who subsequently underwent immunotherapy. Thorac Cancer. 2021, 12(9): 1366-1372.

47. Masatoshi Hanada, Kouta Yamauchi, Shinjiro Miyazaki, et al. Geriatric nutritional risk index, a predictive assessment tool, for postoperative complications after abdominal surgery: A prospective multicenter cohort study. Geriatr Gerontol Int. 2019, 19(9): 924-929.

48. Xie H, Tang S, Wei L, Gan J. Geriatric nutritional risk index as a predictor of complications and longterm outcomes in patients with gastrointestinal malignancy: a systematic review and meta-analysis. Cancer Cell Int. 2020, 20(1): 530.

\section{Tables}

Table 1. Patient characteristics 


\begin{tabular}{ll} 
Variable & Number (percent) \\
\hline Total & 128 \\
\hline Age, mean & 65.77 \\
\hline Sex & $94(73.43 \%)$ \\
\hline Male & $34(26.57 \%)$ \\
\hline Female & \\
\hline Surgery method & $120(93.75 \%)$ \\
\hline Thoracotomy & $8(6.25 \%)$ \\
\hline VATS & \\
\hline Smoking history & $58(45.31 \%)$ \\
\hline Never & $52(40.63 \%)$ \\
\hline Current & $18(14.06 \%)$ \\
\hline Ever & \\
\hline Pathology & $45(35.16 \%)$ \\
\hline Squamous & $70(54.69 \%)$ \\
\hline Adenocarcinoma & $6(4.69 \%)$ \\
\hline Large cell lung cancer & $2(1.56 \%)$ \\
\hline Small cell lung cancer & $5(3.90 \%)$ \\
\hline Other & 106.91 \\
\hline GNRI, mean & 23.92 \\
\hline BMl, mean & \\
\hline
\end{tabular}

VATS: video-assisted thoracoscope; GNRI: geriatric nutritional risk index; BMI: body mass index.

Table 2. Postoperative pulmonary complications in the study population 


\begin{tabular}{ll} 
Item & 37 \\
\hline Total events & 10 \\
\hline Pneumonia & 2 \\
\hline Atelectasis & 3 \\
\hline Mechanical ventilation $>48 \mathrm{~h}$ & 1 \\
\hline Re-intubation & 19 \\
\hline Persistent air leakage or pleural effusion & 1 \\
\hline Pneumorrhagia & 1 \\
\hline Pulmonary embolism & 29 \\
\hline Total patients & 23 \\
\hline 1 event & 6
\end{tabular}

Table 3. Correlation between postoperative pulmonary complications and immunonutritional parameters 


\begin{tabular}{|c|c|c|c|}
\hline Variable & Non-PPC & PPC & $P$ value \\
\hline \multicolumn{4}{|l|}{ Sex } \\
\hline Male & 67 & 27 & 0.007 \\
\hline Female & 32 & 2 & \\
\hline Age, years & $65.98 \pm 4.95$ & $65.07 \pm 3.36$ & 0.354 \\
\hline Surgery method & & & 0.682 \\
\hline VATS & 7 & 1 & \\
\hline Thoracotomy & 92 & 28 & \\
\hline Smoking history & & & 0.609 \\
\hline Never & 47 & 11 & \\
\hline Current & 38 & 14 & \\
\hline Ever & 14 & 4 & \\
\hline Pathology & & & 0.052 \\
\hline Squamous & 28 & 17 & \\
\hline Adenocarcinoma & 60 & 10 & \\
\hline Small cell lung cancer & 2 & 0 & \\
\hline Large cell lung cancer & 5 & 1 & \\
\hline Other & 4 & 1 & \\
\hline GNRI & $107.85 \pm 9.37$ & $103.69 \pm 9.38$ & 0.038 \\
\hline BMI & $24.21 \pm 3.33$ & $22.94 \pm 2.75$ & 0.065 \\
\hline FEV1\% & $85.37 \pm 16.71$ & $74.83 \pm 18.13$ & 0.004 \\
\hline FVC\% & $86.94 \pm 15.44$ & $81.09 \pm 14.23$ & 0.070 \\
\hline LY & $1.87 \pm 0.57$ & $1.75 \pm 0.54$ & 0.287 \\
\hline LY\% & $30.71 \pm 7.98$ & $24.73 \pm 9.10$ & 0.001 \\
\hline RBC & $4.37 \pm 0.50$ & $4.33 \pm 0.52$ & 0.700 \\
\hline HB & $132.80 \pm 14.22$ & $134.52 \pm 14.79$ & 0.571 \\
\hline ALB & $42.06 \pm 3.75$ & $41.03 \pm 4.39$ & 0.213 \\
\hline PALB & $0.22 \pm 0.06$ & $0.21 \pm 0.06$ & 0.407 \\
\hline Globin & $26.68 \pm 4.20$ & $25.69 \pm 3.32$ & 0.246 \\
\hline
\end{tabular}




\begin{tabular}{llll} 
Urea & $5.54 \pm 1.41$ & $5.83 \pm 1.90$ & 0.366 \\
\hline Creatinine & $62.72 \pm 10.92$ & $65.45 \pm 15.92$ & 0.291 \\
\hline Uric acid & $321.60 \pm 70.54$ & $309.93 \pm 84.38$ & 0.456 \\
\hline Number of segments removed & $4.13 \pm 1.69$ & $4.24 \pm 1.55$ & 0.754
\end{tabular}

Data represent mean \pm standard deviation. Abbreviations: PPC, post pulmonary complication; VATS: video-assisted thoracoscope; GNRI, geriatric nutritional risk index; BMI: body mass index; FEV1: forced expiratory volume; FVC: forced vital capacity; LY: lymphocyte; RBC: red blood cell; HB: hemoglobin; ALB: albumin; PALB: Prealbumin.

Table 4. Receiver operating characteristic curve analysis of immunonutritional parameters

\begin{tabular}{lllllll} 
Parameter & AUC & P value & Cutoff value & Sensitivity & Specificity & Youden index \\
\hline GNRI & 0.639 & 0.023 & 105.736 & 0.646 & 0.655 & 1.301 \\
\hline BMI & 0.599 & 0.104 & & & & \\
\hline FEV1 & 0.664 & 0.007 & 77.150 & 0.657 & 0.655 & 1.312 \\
\hline FVC & 0.603 & 0.093 & 89.05 & 0.424 & 0.793 & 1.217 \\
\hline LY\% & 0.686 & 0.002 & 27.300 & 0.657 & 0.655 & 1.312
\end{tabular}

Abbreviations: GNRI, geriatric nutritional risk index; BMI: body mass index; FEV1: forced expiratory volume; FVC: forced vital capacity; LY: lymphocyte; AUC: area under the curve.

Table 5: Multivariate analysis of the influence parameters of PPCs

\begin{tabular}{llll} 
Parameters & OR & $95 \% \mathrm{Cl}$ & P value \\
\hline GNRI: High Vs Low & 0.377 & $0.145,0.975$ & 0.044 \\
\hline Sex冈female vs male & 0.221 & $0.016,1.066$ & 0.060 \\
\hline LY\%: High Vs Low & 0.390 & $0.152,1.002$ & 0.051 \\
\hline FEV1\%: High Vs Low & 0.296 & $0.116,0.759$ & 0.011
\end{tabular}

Abbreviations: GNRI, geriatric nutritional risk index; BMI: body mass index; FEV1: forced expiratory volume; LY: lymphocyte; OR: odd ration; Cl: confidence interval.

\section{Figures}




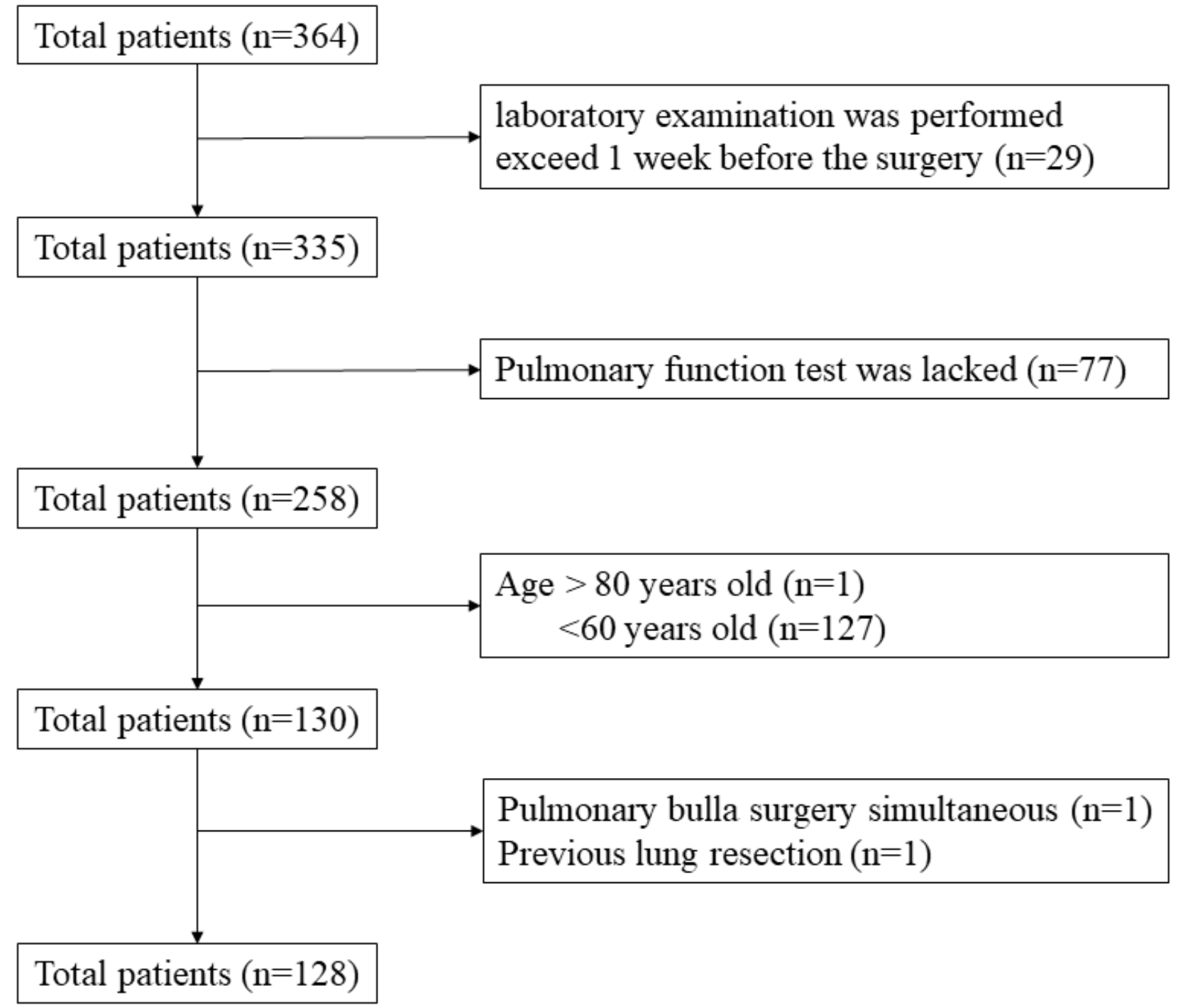

Figure 1

Patient selection flow chart 


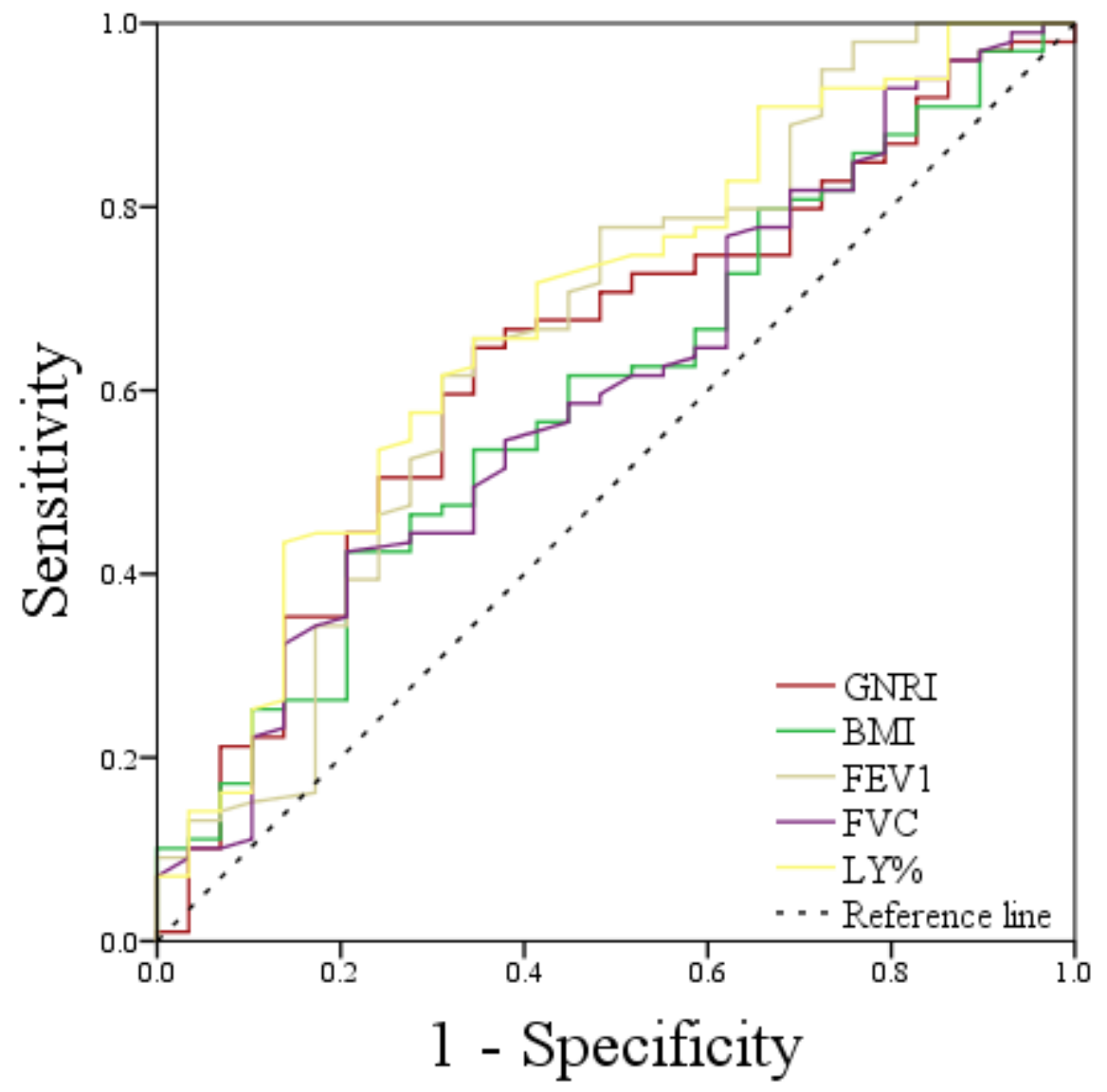

Figure 2

ROC for the patients' characteristics. Abbreviation: GNRI, geriatric nutritional risk index; BMI: body mass index; FEV1: forced expiratory volume; FVC: forced vital capacity; LY: lymphocyte; AUC: area under the curve. 